\title{
Accelerated diagnostic protocol for patients with chest pain
} assessed in a randomized clinical trial

An accelerated diagnostic protocol (ADP) for patients presenting to an emergency department (ED) with chest pain has been compared with a conventional diagnostic protocol in a single-centre, randomized clinical trial. The investigators believe that their trial "is an important first step in proving that [use of the ADP] is feasible and will facilitate early discharge from the ED".

ED overcrowding is a common problem worldwide, and is associated with adverse patient outcomes. Whilst large numbers of patients is an obvious cause of ED overcrowding, prolonged patient observation is also a contributing factor. Notably, a substantial proportion of patients presenting to EDs have chest pain suggestive of an acute coronary syndrome. Incorrect diagnoses can have dire consequences for these individuals, but a reliable ADP to identify low-risk patients could be of benefit not only to those with chest pain, but also to other patients who need access to ED resources.
In total, 542 patients presenting to the ED with chest pain were randomly assigned to undergo either the $2 \mathrm{~h} \mathrm{ADP}$ or a conventional diagnostic protocol, and then followed up for 30 days. The ADP was associated with a signficantly greater proportion of patients who were discharged within $6 \mathrm{~h}$ of presentation and experienced no major adverse cardiac events (MACE)during follow-up (19.3\% vs $11.0 \%$ with the conventional protocol; OR 1.92, 95\% CI 1.18-3.13, $P=0.008$ ). The number of MACE did not differ significantly between the two groups.

Of note, an additional 35 patients in the ADP group (13\%) were identified as being at low risk, but were admitted to the hospital anyway. None of these patients experienced MACE. The trial investigators, therefore, postulate that "higher early discharge might be achievable with greater acceptance of the protocol by clinicians."

Bryony M. Mearns

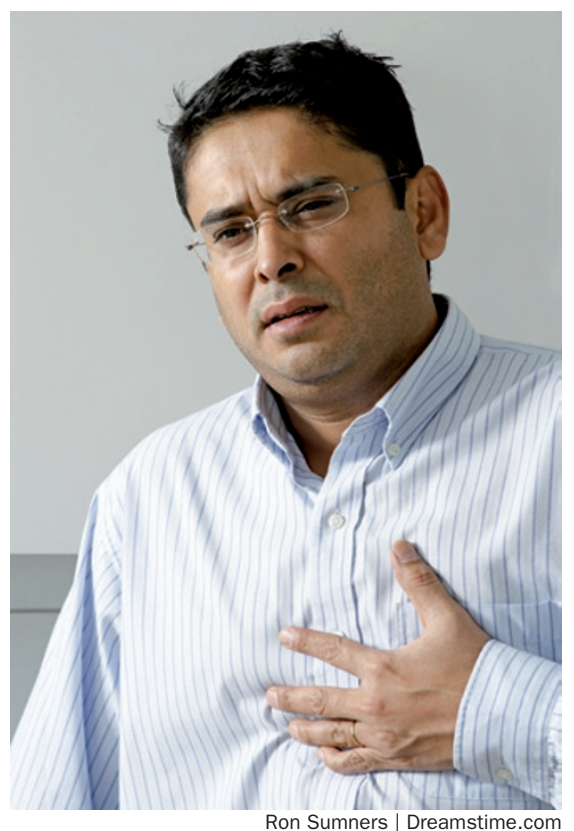

Original article Than, M. et al. A 2-hour diagnostic protocol for possible cardiac chest pain in the emergency department: a randomized clinical trial. JAMA Intern. Med. doi:10.1001/jamainternmed.2013.11362 